\title{
Microwave Detection of Longitudinal Crack and Identification of Its Location in Straight Pipe*
}

\author{
Kavoos ABBASI**, Satoshi ITO** and Hidetoshi HASHIZUME** \\ **Dept. of Quantum Science \& Energy Engineering, Tohoku University \\ Aramaki, Aoba, Sendai, 980-8579, Japan \\ E-mail:abbasi@karma.qse.tohoku.ac.jp
}

\begin{abstract}
A microwave NDT method based on guided electromagnetic wave is described in order to detect longitudinal crack and evaluate its location in steel pipe. To detect longitudinal crack in pipe, suitable guided-mode should be propagated in the inspected pipe with crack. For this purpose, first and dominant circular $\mathrm{TE}_{11}$-mode is sent to the system by using a mode-converter. Mode-converter is used to convert rectangular $\mathrm{TE}_{10}$-mode to the circular $\mathrm{TE}_{11}$-mode. A network analyzer, which is a generator and receiver of the microwaves designed to process the magnitude and phase of the reflected and transmitted waves was used to generate continues signal and to measure the amplitude of reflection coefficient. The goal of this investigation is to demonstrate the capability of this technique for detecting longitudinal cracks in piping system with high speed. More emphasis is put on the evaluation of crack location by measuring time of flight (TOF) of the reflected waves from the crack. The results for two crack locations at several plunger positions either in frequency domain or time domain for measuring TOF of the waves are presented. The experimental results of TOF are compared with calculations to show that by knowing TOF of the reflected wave, it is possible to predict crack locations. The evaluated results of TOFs are shown to agree well with the calculated ones and crack locations can be estimated with error less than $0.13 \%$.
\end{abstract}

Key words: Electromagnetic Waves, Longitudinal Crack, Time of Flight (TOF), Inverse Fast Fourier Transform (IFFT)

\section{Introduction}

The steam generator tubing and piping system of pressurized water reactors must be routinely inspected to guarantee the safety of operations. Defects in these systems should be detected rapidly and accurately in early stages. At present, typical methods for detecting defects are eddy current testing (ECT) and ultrasonic testing (UT). Even though these methods have high accuracy, they are time/cost-consuming for the purpose of the long range inspection of piping. Although the ultrasonic guided wave is also used for rapid inspection of piping system, there is difficult to excite suitable mode for defect detection and sometime interference of several mode makes detection very difficult ${ }^{(1)}$. Hence it is desirable to develop another high speed technique for measuring crack location.

Until 1992, microwave nondestructive techniques had been proposed for detecting surface breaking cracks in metals but only limited success had been achieved in those studies $^{(2)-(5)}$. Since 1992, microwave crack detection techniques that use open ended rectangular waveguides have been developed by Yeh and Huber ${ }^{(6)(9)}$. The previous work on 
Microwave NDT method shows that this technique has potential for inspection of large area in short time.

Sugawara and Hashizume showed that the NDT method using microwaves has strong possibility to detect crack in the straight pipes ${ }^{(10)(11)}$. Due to propagation of electromagnetic guided waves above cutoff frequencies in larger distances in waveguide, without significant attenuation loss, this technique may be suitable for long pipe inspection.

The capability of this method to detect semi circumferential open crack and to predict of crack location was confirmed experimentally ${ }^{(12)}$. In this study some experiments are carried out to detect longitudinal cracks and to predict its location in straight pipe.

\section{Theoretical Background and Experimental Setup}

Since this study is carried out to detect longitudinal cracks in straight pipe, the circular $\mathrm{TE}_{11}$-mode which is suitable for detection of longitudinal cracks should be propagated in straight pipe with cracks. The TE-mode are characterized by fields with $E_{\mathrm{z}}=0$ while $H_{\mathrm{z}} \neq 0$, where $z$ indicates the direction of propagation (In the case of cylindrical pipe, $z$ corresponds to axial direction of the pipe). When an electromagnetic wave is propagated in the waveguide, the electric surface current is produced in the inner surface of the pipe, which can be expressed according to the Ampere's low as the following equation;

$$
n \times\left(H_{2 z}-H_{1 z}\right)=J_{s} \varphi_{0}
$$

where $n$ and $\varphi_{0}$ are the unit vectors in radial and circumferential directions, respectively. $H_{1 z}$ and $H_{2 z}$ are the z-component of the magnetic field in tow media, air (empty space in the pipe) and conductor, respectively and $J_{\mathrm{s}}$ is the surface current density. When this surface current flows in the straight pipe with a longitudinal crack, the crack prevents the surface current from flowing. Change in the surface currents makes change in the electromagnetic wave and consequently some parts of incident wave are reflected and reflected wave has information related to the crack.

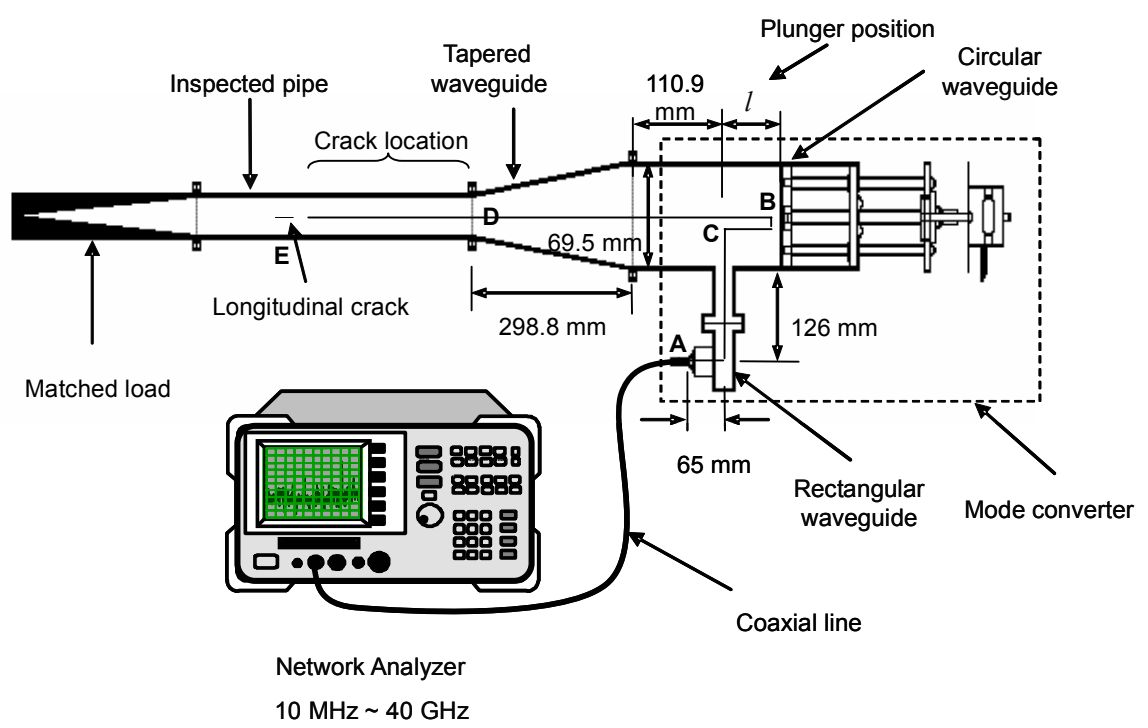

Fig.1 Experimental system for detection of longitudinal crack in straight pipe 
A schematic diagram of the experimental setup is given in Fig.1. The electromagnetic wave, which is generated by network analyzer, passes through the mode converter via the coaxial line. The mode converter is consist of rectangular waveguide (C-band) and circular waveguide and converts the rectangular TE-modes to the circular TE and TM-modes.

To reserve TE-modes and cancel TM-modes, the plunger is mounted in the circular waveguide. The first dominant circular TE-mode is $\mathrm{TE}_{11}$-mode This mode can be resonated in the system by moving the plunger in the circular waveguide. The conditions to resonate or damp the modes are given by the following equations;

$$
\begin{array}{ll}
l=\lambda_{\mathrm{g}} \cdot \frac{m}{2} & \text { (for resonating) } \\
l=\lambda_{\mathrm{g}} \cdot \frac{(2 m+1)}{4} & \text { (for damping) }
\end{array}
$$

where $l$ shows the distance between plunger and center of the rectangular waveguide and $m$ is an integer. The $\lambda_{\mathrm{g}}$ denotes guided wavelength and can be obtained by the following equation in term of frequency.

$$
\lambda_{\mathrm{g}}=\frac{\lambda}{\sqrt{1-\left(\frac{f_{c}}{f}\right)^{2}}}
$$

where $f_{\mathrm{c}}$ is the cutoff frequency of the waveguide. Group velocity of the wave, which is important parameter for calculating TOF or crack locations, is also given by the following equation.

$v_{\mathrm{g}}=\frac{c}{\sqrt{\mu_{\mathrm{R}} \varepsilon_{\mathrm{R}}}} \sqrt{1-\left(\frac{f_{\mathrm{c}}}{f}\right)^{2}}$

where $c, \mu_{\mathrm{R}}, \varepsilon_{\mathrm{R}}$ are light velocity, relative permeability and relative permittivity, respectively.

The test pipe made of type 304 stainless steel, has an inner diameter of $34 \mathrm{~mm}$, outer diameter of $38 \mathrm{~mm}$ and thickness of $2 \mathrm{~mm}$. The cutoff frequency of this pipe for $\mathrm{TE}_{11}$-mode is $5.17 \mathrm{GHz}$. The crack is a longitudinal slit whose length is $40 \mathrm{~mm}$ and width is $0.3 \mathrm{~mm}$ and made by rotating blade from the outer surface of the pipe to the inner surface as seen in Fig. 2. To reduce environmental noise and absorbing transmitted wave, the matched load made of paraffin and graphite, is mounted at the end of the pipe. The electromagnetic modes can be resonated or reduced by changing plunger position from $90 \mathrm{~mm}$ (minimum position) to $180 \mathrm{~mm}$ (maximum position). The experiment was performed for pipes without fluid and can also be done for water filled pipe but due to high loss of electromagnetic wave in water

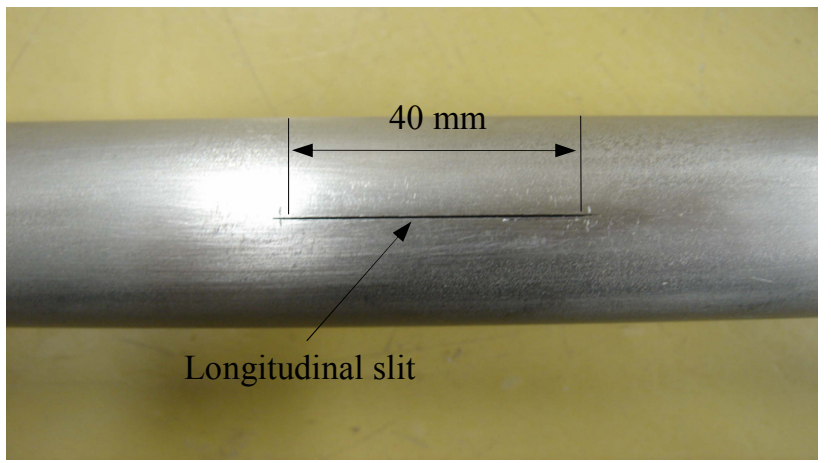

Fig.2 Pipe with longitudinal slit 
at high frequencies it is probable that the inspection length reduces relative to pipes without waters.

\section{Results and Discussions}

The experiment was carried out for two crack locations of $400 \mathrm{~mm}$ and $800 \mathrm{~mm}$. The crack location is defined in Fig.1. Because electromagnetic waves are propagated with frequencies above cutoff frequency, frequency range of $5.2 \mathrm{GHz} \sim 6 \mathrm{GHz}$ was chosen in this experiment. This frequency range is chosen based on the cutoff frequency of circular $\mathrm{TE}_{11}$-mode $(5.17 \mathrm{GHz})$ and cutoff frequency of next circular $\mathrm{TM}_{01}$-mode $(6.758 \mathrm{GHz})$, in order to pass only $\mathrm{TE}_{11}$-mode in the system. The result shows that the frequency range of $6 \sim 6.758 \mathrm{GHz}$ has not significant effect on the reflected signal. Hence maximum frequency of $6 \mathrm{GHz}$ is selected in this study. Fig. 3 and Fig. 4 show the results for crack located at 400 $\mathrm{mm}$ in frequency domain and time domain, respectively. Fig. 5 and Fig. 6 show the same results for crack located at $800 \mathrm{~mm}$ when plunger position is changed from $90 \mathrm{~mm}$ to 180 $\mathrm{mm}$. These results are obtained by subtraction of reflection coefficient $(I)$ of two signals of pipe with and without crack. Reflection coefficient is defined as the ratio of the reflected signal voltage to the incident signal voltage. The difference $\Delta \Gamma$ defined by the following equation.

$\Delta \Gamma=\Gamma_{2}-\Gamma_{1}$

where $\Gamma_{1}$ and $\Gamma_{2}$ are $\Gamma$ in the case without crack and with crack respectively. Fig.3 and Fig.5 show that the large difference between two signals (with and without crack) has been observed in frequency range of $5.2 \mathrm{GHz}$ to $5.6 \mathrm{GHz}$.

Additional information about the crack and crack location has been obtained by considering these differences of two signals in the time domain by getting Inverse Fast Fourier Transform (IFFT) of the signal in the frequency domain. Fig.4 and Fig.6 clearly show the results for crack location of $400 \mathrm{~mm}$ and $800 \mathrm{~mm}$ in this case, respectively. As these figures show, the large difference of two signals occurs at time of about $11 \mathrm{~ns}$ for crack located at $400 \mathrm{~mm}$ and $16 \mathrm{~ns}$ for crack located at $800 \mathrm{~mm}$. In these figures the dashed lines indicates the TOFs obtained by the calculation.

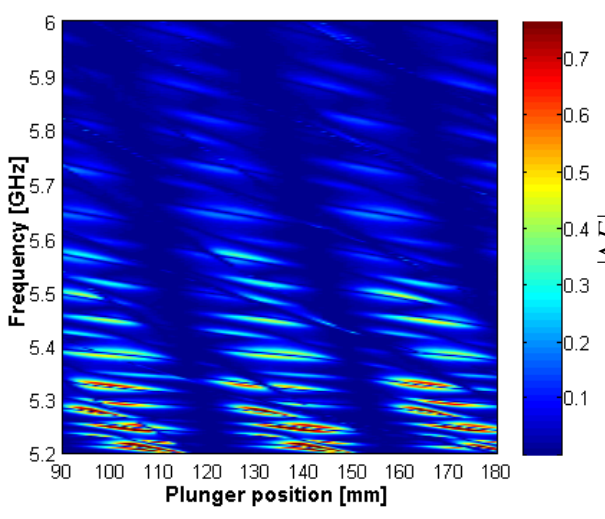

Fig.3 Change of $\Delta \Gamma$ in frequency domain for crack located at $400 \mathrm{~mm}$ at different plunger position

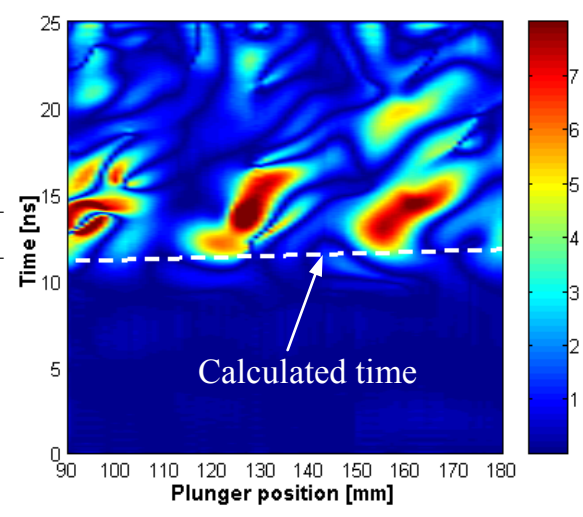

Fig.4 Change of $\Delta \Gamma$ in time domain for crack located at $400 \mathrm{~mm}$ at different plunger position 
Calculated times $(t)$ can be obtained with knowing group velocity and traveling path of the electromagnetic waves according to the following equation.

$t=\frac{\text { (Traveling path of the electromagnetic waves) }}{v_{\mathrm{g}}}$

The total path of traveling wave is shown in Fig. 1 as A-B-E-B-A. It is seen from equation (5) that group velocity of the waves increases with increasing frequency and consequently time of flight decreases for specific traveling path.

These experimental results show a good agreement with the calculated ones. In these figures, it can be also seen that the large differences between two signals occurs in specific plunger positions. This means that signal are resonated in mode converter by changing the plunger position and there is the dependency of generated mode in the system on the plunger position.

Fig. 4 and Fig. 6 show that the minimum TOF (the time of the first significant peak) can be estimated by the experimental result. As mentioned above, the minimum TOF is related to the maximum frequency in the given frequency range $(5.2 \sim 6 \mathrm{GHz})$. Therefore the

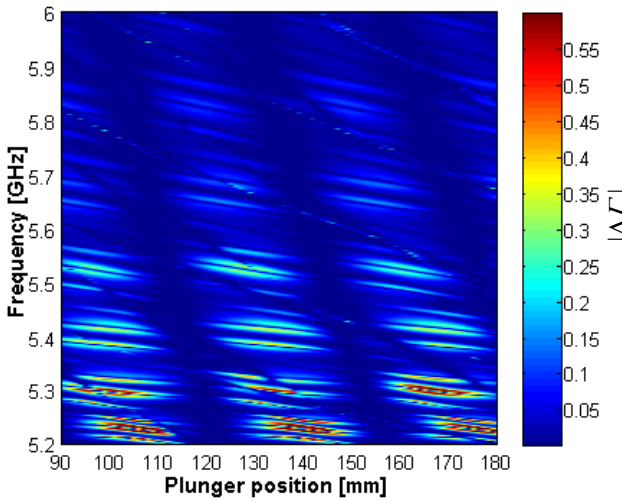

Fig.5 Change of $\Delta \Gamma$ in frequency domain for crack located at $800 \mathrm{~mm}$ at different plunger position

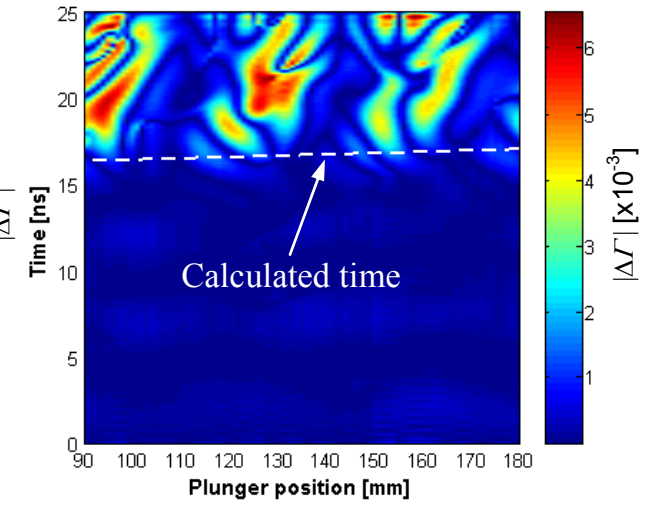

Fig.6 Change of $\Delta \Gamma$ in time domain for crack located at $800 \mathrm{~mm}$ at different plunger position

calculations of TOFs are performed at frequency of $6 \mathrm{GHz}$. As it can be seen from the Fig. 4 and Fig. 6 there are some signal with larger TOFs (times after the arrival time of the first significant peak). These signals have frequency lower than the maximum frequency in the given frequency range.

From the above results in Fig. 4 and Fig. 6, it can be seen that large response of the electromagnetic waves occurs in several plunger positions such as $90 \mathrm{~mm}, 120 \mathrm{~mm}$ and 160 $\mathrm{mm}$. As an example, the result when plunger located at $120 \mathrm{~mm}$ is given in Fig. 7. As this figure show, the response of electromagnetic wave to the crack is clearly distinct in time domain signals. In this figure the calculated time which specified by solid arrows are 11.08 ns and $16.34 \mathrm{~ns}$ for crack located at $400 \mathrm{~mm}$ and $800 \mathrm{~mm}$, respectively. It can be seen that these arrows almost coincide to the minimum point before the large peak in the Fig. 7(a) and 7(b). The related time to these minimum points are $10.7 \mathrm{~ns}$ and $16.78 \mathrm{~ns}$ and correspondence crack locations are $351 \mathrm{~mm}$ and $814 \mathrm{~mm}$, respectively. This means that crack location can be predicted with error less than $0.13 \%$. 


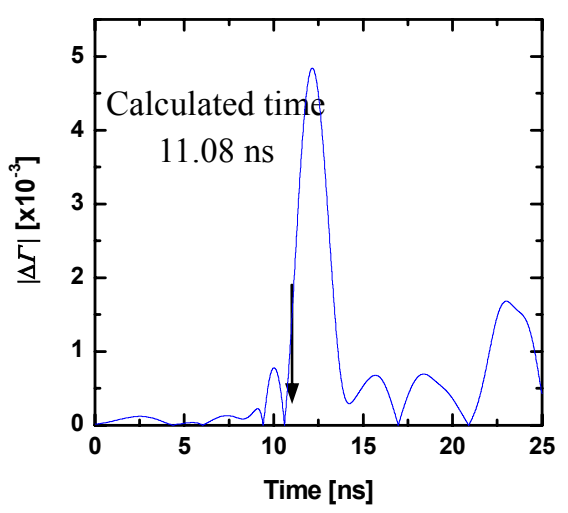

(a)

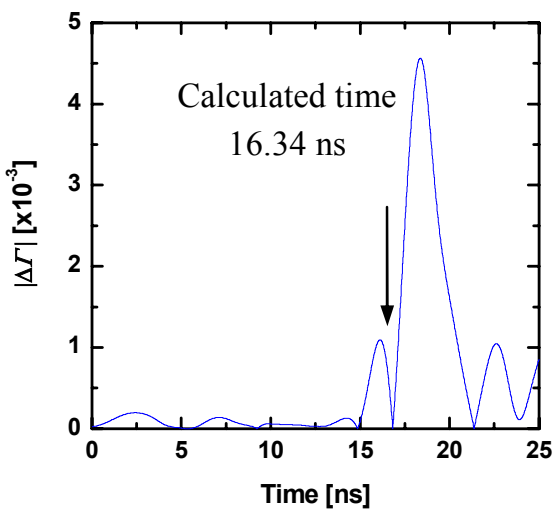

(b)

Fig.7 Change of $\Delta \Gamma$ in time domain for plunger position of $120 \mathrm{~mm}$ (a) crack located at $400 \mathrm{~mm}$ and (b) crack located at $800 \mathrm{~mm}$

\section{Conclusions}

Through this study, NDT method using high frequency electromagnetic waves to detection of longitudinal crack was demonstrated. The results showed that $\mathrm{TE}_{11}$-mode was resonated in the system by changing plunger location. To get information about crack and crack location, the results were shown in both time domain and frequency domain. By considering signal in time domain which obtained by getting IFFT of the signal in frequency domain, the result gave good information about the TOF and there was good agreement between calculated TOF and experimental result. With knowing TOF, crack location can be predicted by this method clearly.

The crack with length of $40 \mathrm{~mm}$ was investigated in this study. For next experiments cracks with shorter length in the longer pipe will be investigated to show detection limit of this method. Because of limitation of making crack width smaller than $0.3 \mathrm{~mm}$, the minimum width of crack that can be detected by this technique should be investigated with numerical analysis. The detection of crack in bend pipe and piping system as an application of this technique will be investigated in the next experiment.

\section{References}

(1)Cheong, Y. M, et al., Ultrasonics vol.42, (2004), pp. 883-888

(2)Bahr, A. J, ASTM STP 722, G. Birnbaum and G. Free, eds., American Society for Testing and aterials, Philadelphia, (1981), pp. 311-331.

(3)Auld, B. A, ASTM STP 722, G. Birnbaum and G. Free, eds., American Society for Testing and Materials, Philadelphia, (1981), pp. 332-347.

(4)Auld, B. A., 1978, G.L. Report 2839, E.L. Ginzton Laboratory, Stanford University, Stanford, CA, July.

(5)Feinstein, L., and Hruby, R.J., In: 6th Symp. on Nondestructive Evaluation of Aerospace and Weapons Systems Components and Materials, San Antonio, TX, 1967.

(6)Yeh, C. and Zoughi, R., IEEE Trans. Instrum. Measure Vol. 43, (1994), Issue 5, pp 719. 
(7) Huber, C., et al., IEEE Trans. Microwave Theory Technique Vol. 45, (1997), Issue 4, pp 477.

(8)Yeh, C., Ranu, E. and Zoughi, R., Mater. Eval. Vol. 52, Issue 6, (1994), pp. 676.

(9)Yeh, C. and Zoughi, R., Res. Nondestr. Eval. Vol.6, Issue1, (1994), pp. 35.

(10)Sugawara, K., Hashizume, H., and Kitagima, S., JSAEM Studies in Applied Electromagnetic and Mechanics Vol.10, (2001), pp. 313-316.

(11)Hashizume, H., et al, eNDE2003, Saclay, Studies in Applied Electromagnetic and Mechanics Vol. 24, (2003), pp 263-270.

(12)Abbasi, K., et al., The $11^{\text {th }}$ International Workshop on Electromagnetic Nondestructive Evaluation, IWATE, Japan, 2006 